INPLASY

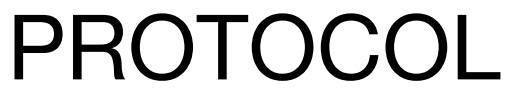

To cite: Chen et al. Effects of Guizhi decoction for diabetic cardiac autonomic neuropathy: a protocol for a systematic review and meta-analysis. Inplasy protocol 202080018. doi:

10.37766/inplasy2020.8.0018

Received: 05 August 2020

Published: 05 August 2020

Corresponding author: Junmin Chen

1335085129@qq.com

Author Affiliation:

Hospital of Chengdu

University of TCM

Support: National key R \& D plan

Review Stage at time of this submission: The review has not yet started.

Conflicts of interest:

None.

\section{Effects of Guizhi decoction for diabetic cardiac autonomic neuropathy: a protocol for a systematic review and meta-analysis}

Chen, JM1; Cai, JW2; Wei, MY3; Zhang, XY4; Zhong, M5; Liu, M6; Chen, $Q^{7}$.

Review question / Objective: How effective is Guizhi decoction supplementation in the treatment of diabetic cardiac autonomic neuropathy?

Condition being studied: Guizhi decoction.diabetic cardiac autonomic neuropathy.

Information sources: The English literature mainly searches Cochrane Library, PubMed, EMBASE, and Web of Science, while the Chinese literature comes from C N K I , C B M , V I P, a $n d \mathbf{~} \mathbf{~ a ~} n g$ f a $n g$ database.Simultaneously we will retrieval clinical registration tests and grey literatures.

INPLASY registration number: This protocol was registered with the International Platform of Registered Systematic Review and Meta-Analysis Protocols (INPLASY) on 05 August 2020 and was last updated on 05 August 2020 (registration number INPLASY202080018).

\section{INTRODUCTION}

Review question / Objective: How effective is Guizhi decoction supplementation in the treatment of diabetic cardiac autonomic neuropathy?
Condition being studied: Guizhi decoction.diabetic cardiac autonomic neuropathy.

\section{METHODS}

Participant or population: a) DCAN patients must meet the World Health Organization (WHO) "diabetes" diagnosis standard in 
1999. b)The diagnosis standard of cardiac autonomic neuropathy shall refer to Toronto standard and European cardiovascular disease prevention guidelines, regardless of race, gender and age. c) Special patients with severe diabetic complications, severe cardiac insufficiency, pregnancy, etc. are not included.

Intervention: This meta-analysis will include the RCTs of Guizhi decoction supplementation regardless of dose and frequency. Trials will be included at least 8 weeks of treatment.

Comparator: This meta-analysis will include the RCTs that administered with placebo, that with no treatment or active pharmacological treatment (regardless of dose, frequency).Trials with a minimum treatment duration of at least 8 weeks will be included.

Study designs to be included: We will include randomised trials to assess the beneficial effects of the treatments, and will supplement these with observational studies.

Eligibility criteria: The diagnosis standard of cardiac autonomic neuropathy shall refer to Toronto standard and European cardiovascular disease prevention guidelines, regardless of race, gender and age.

Information sources: The English literature mainly searches Cochrane Library, PubMed, EMBASE, and Web of Science, while the Chinese literature comes from C N K I , C B M , V I P, a n d W a n g f a n g database.Simultaneously we will retrieval clinical registration tests and grey literatures.

Main outcome(s): The primary outcomes include patient before and after treatment: markedly effective: symptoms improved significantly > 70\%; effective: symptoms reduced by $30 \%$ to $70 \%$; ineffective: symptom improvement is $<30 \%$ or no improvement, or even worse. In addition to
Heart rate variability (HRV) recognized as the most accurate and sensitive index to judge whether diabetic patients have autonomic nervous system damage, fasting blood glucose(FBG) and 2 hours Postprandial Blood Glucose (2 h PBG) were also included. Secondary outcomes included glycosylated hemoglobin and adverse events.

Quality assessment / Risk of bias analysis: Assessing the risk of bias in studies. In this tool, the risk of bias of a trial is assessed through 7 items: random sequence generation (selection bias), allocation concealment (selection bias), blinding of participants and personnel (performance bias), blinding of outcome assessment (detection bias), incomplete outcome data (attrition bias), selective reporting(reporting bias), other bias. Each item is classified as "Low risk","High risk" or "Unclear risk". Two reviewers will conduct the risk of bias assessment independently and any disagreements will be solved by a discussion of all reviewers.

Strategy of data synthesis: Substantial when P50\%. If P>.05 and $\mathrm{I}^{2}<50 \%$, then the studies included is homogeneous and the differences between them can be ignored. If there is significant heterogeneity, the random effect model will used to pool data, and if there is no significant heterogeneity, then the fixed effect model will be used. If quantitative synthesis is not appropriate due to substantial heterogeneity, then the results will be presented with tables and figures.

Subgroup analysis: Substantial when $\mathrm{P} 50 \%$. If $\mathrm{P}>\mathbf{0 5}$ and $\mathrm{I}^{2}<50 \%$, then the studies included is homogeneous and the differences between them can be ignored. If there is significant heterogeneity, the random effect model will used to pool data, and if there is no significant heterogeneity, then the fixed effect model will be used. If quantitative synthesis is not appropriate due to substantial heterogeneity, then the results will be presented with tables and figures. 
Sensibility analysis: Sensitivity analysis is mainly used to evaluate the robustness of the primary outcome measures. The method is that removing the low-level quality study one by one and then merge the data to assess the impact of sample size, study quality, statistical method, and missing data on results of metaanalysis.

Country(ies) involved: China.

Keywords: Guizhi decoction, diabetic cardiac autonomic neuropathy, protocol, systematic review, meta-analysis.

Contributions of each author:

Author 1 - Junmin Chen.

Author 2 - Jiawei Cai.

Author 3 - Mengya Wei.

Author 4 - Xiaoran Zhang.

Author 5 - Min Zhong.

Author 6 - Min Liu.

Author 7 - Qiu Chen. 